\title{
Family History of Dementia Predicts Worse Neuropsychological Functioning Among HIV Infected Persons
}

\author{
David J. Moore, Ph.D. ${ }^{1,2}$, Miguel Arce, B.A. ${ }^{1,2}$, Suzanne Moseley, B.S. ${ }^{1}$, J. Allen McCutchan, \\ M.D. ${ }^{1,2,3}$, Jennifer Marquie-Beck, M.P.H. ${ }^{1,2}$, Donald R. Franklin, B.S. ${ }^{1,2}$, Florin Vaida, Ph.D. \\ ${ }^{2,4}$, Cristian L. Achim, M.D., Ph.D. ${ }^{1}$, Justin McArthur, M.B.B.S., M.P.H. ${ }^{5}$, Susan Morgello, \\ M.D. ${ }^{6}$, David M. Simpson, M.D. ${ }^{6}$, Benjamin B. Gelman, M.D., Ph.D. ${ }^{7}$, Ann C. Collier, M.D. ${ }^{8}$, \\ Christina M. Marra, M.D. ${ }^{8}$, David B. Clifford, M.D. ${ }^{9}$, Robert K. Heaton, Ph.D. ${ }^{1,2}$, Igor Grant, \\ M.D. ${ }^{1,2}$, and the CHARTER Group, and the HNRC Group \\ ${ }^{1}$ University of California, San Diego (UCSD), Department of Psychiatry \\ ${ }^{2}$ UCSD, HIV Neurobehavioral Research Center (HNRC) \\ ${ }^{3}$ UCSD, Department of Medicine \\ ${ }^{4}$ UCSD, UCSD Division of Biostatistics and Bioinformatics \\ 5Johns Hopkins University; Baltimore, MD \\ ${ }^{6}$ Mount Sinai School of Medicine; New York, NY \\ ${ }^{7}$ University of Texas Medical Branch; Galveston, TX \\ ${ }^{8}$ University of Washington, Seattle; Seattle, WA \\ ${ }^{9}$ Washington University, St. Louis; St. Louis, MO
}

\section{Abstract}

HIV-negative individuals with a family history of dementia (FHD) are more likely to develop dementia than those without a FHD. Whether FHD increases risk for neuropsychological (NP) impairment in HIV+ persons is unknown. As part of a multi-site study into HIV-Associated Neurocognitive Disorders (HAND), we captured FHD with a free-response, self-report question, and assessed NP performance with a comprehensive battery of tests. We examined HIV+ persons with ( $n=190)$ and without ( $n=916)$ self-reported FHD. Despite the fact that the FHD group had factors typically associated with better NP performance (e.g., higher CD4 counts and estimated verbal IQ [VIQ]), persons with FHD had significantly worse NP ability than those without FHD as measured by a Global Deficit Score (GDS) (FHD mean=0.66; No FHD mean=0.55; $\mathrm{p}<0.05$ ). Thus, FHD appears to be a risk factor for HAND; the mechanism(s) underlying how FHD contributes to NP impairment among HIV+ persons warrants study.

\section{Keywords}

HIV; AIDS; Cognition; Aging; Dementia

Location of Work and Address for Reprints: David J. Moore, Ph.D., HIV Neurobehavioral Research Center (HNRC), University of California, San Diego, Department of Psychiatry, 220 Dickinson Street, Suite B, Mail Code 8231, San Diego, CA 92103-8231, (619) 543-5093, FAX (619) 543-1235, djmoore@ucsd.edu. 


\section{Background}

Since the introduction of highly-active antiretroviral therapy (ART) the population of HIV infected individuals has become older because AIDS related mortality has decreased substantially [1]. Currently, $11 \%$ of AIDS patients are living beyond 50 years [2] and by 2015, it is estimated that more than half of HIV+ persons in the U.S. will be over 50 [3]. ART has had a positive effect on expected lifespan and has also decreased the proportion of individuals with HIV-associated dementia (HAD), the most severe form of HIV-associated Neurocognitive Disorders (HAND) [4-8]. Despite the decline in HAD, the prevalence of milder forms of HAND (e.g., Asymptomatic Neurocognitive Impairment (ANI) and Minor Neurocognitive Disorder (MND)) remains high [4, 7, 9, 10]. In addition, as people live longer with HIV/AIDS, the risk of developing neuropsychological (NP) difficulties associated with traditional neurodegenerative disorders (e.g., Alzheimer's disease) and/or the combination of neurodegenerative disorders and HIV increases [2, 8].

Neuropathologic markers, common in patients with neurodegenerative disorders, are more prevalent among older persons with HIV infection as compared to those without HIV infection [11-14]. Specifically, Green et al. [13] found evidence of $\beta$-amyloid deposition in the frontal cortex of approximately $50 \%$ of HIV+ cases in a cohort that had a mean age of 44 , predominantly in the neuronal soma and axonal processes; some cases had extracellular plaques and a few demonstrated deposition in blood vessels. Achim et al. [11] also found an accumulation of $\beta$-amyloid in HIV infected persons ranging from 38-60 years old. These findings suggest that longer life expectancy with HIV could increase pathological aggregation of neuronal proteins that are associated with NP impairment. Another central nervous system (CNS) complication that is associated with aging is reduction of cerebral gray matter volume and neuronal density [15]. Studies using Magnetic Resonance Spectroscopy (MRS) have also revealed neuronal damage in the frontal white matter and the basal ganglia of older HIV patients as compared to seronegative controls [16].

HIV-induced damage to neurons is most likely mediated through indirect neurotoxic mechanisms and is associated with neurocognitive impairment [17-19]. This neuron toxicity can cause synaptodendritic damage, neuronal loss, astrocytosis, and microgliosis that has been shown to be associated with HIV-associated neurocognitive disorders [20-23].

Whether other age-related diseases and HIV combine to cause greater than anticipated NP impairment is not entirely clear [2]. Some studies have shown that HIV infected individuals over the age of fifty have a significantly higher prevalence of HAND compared to younger individuals [24-28]. For example, age and HIV both contributed to impairment in eventbased prospective memory (remembering to remember) even after controlling for possible confounding demographic, medical, and psychiatric factors [29]. Additive cognitive effects of aging and HIV have potential clinical and public health consequences since cognitive impairment contributes to poor adherence to ART [29-31].

Neurodegenerative diseases such as Alzheimer's disease (AD) are thought to be at least partially inherited; for example, apolipoprotein E4 (APOE4) allele increases risk for AD [32-35] and is associated with worse neurocognitive outcomes in those without AD [36-38]. As in AD, HIV subjects with at least one copy of the APOE4 allele have increased risk of developing HIV-associated dementia suggesting that APOE4 increases HIV-induced damage to the brain [26].

Capturing family history of dementia (FHD) via self-report is an inexpensive proxy for genetic markers known to increase risk of dementia. An advantage of FHD is that it may detect familial neurodegenerative conditions for which genetic correlates or screenings are not available. Several studies have shown that simply having FHD places one at a higher 
risk of developing $\mathrm{AD}$ [39-41]. For example, one study showed that offspring of parents that both developed $\mathrm{AD}$ are at an even higher risk of developing $\mathrm{AD}$ themselves [39]. Another study established that FHD and age are important risk factors for developing AD [41]. Interestingly, Fox et al [42] showed that persons with FHD show a measurable cognitive decline years before the onset of AD. Furthermore, studies have suggested an additive effect of FHD and APOE4 on age of dementia onset, risk of dementia development, and rate of cognitive decline [43-45].

Since no studies have specifically reported FHD as a potential risk factor for HIV-associated neurocognitive disorders, we determined if NP functioning was worse among HIV-infected persons with a family history of dementia as compared to those without such a history. If FHD is associated with NP impairment, we wanted to assess its strength in the context of other factors (e.g., HIV disease severity) known to be associated with HIV-associated neurocognitive impairment.

\section{Participants and Methods}

\section{Subjects}

We evaluated 1106 HIV seropositive (HIV+) men and women who were participating in the multi-site CNS HIV Antiretroviral Therapy Effects Research (CHARTER) study of HAND at 6 US academic medical centers. Family history of dementia (FHD) was captured using a free-response self-report question. Only first-degree (i.e., biologic parent, sibling, or offspring) and second-degree (i.e., biologic grandparent, grandchild, uncle, aunt, nephew, niece, half-sibling) relatives were considered for this study using the following intervieweradministered question:

"Is there a history of neurologic illness in your family such as Parkinson's Disease, Alzheimer's Disease, Huntington's Chorea, Multiple Sclerosis, Epilepsy, etc.? If so, who and was this from maternal or paternal side of the family?"

Because some of the listed neurologic conditions do not necessarily cause dementia symptoms in all those afflicted, we chose to code persons as FHD positive only if they responded that a family member was diagnosed with "dementia" or Alzheimer's Disease (AD). Raters were trained to ask follow-up questions regarding the type of dementia, the specific afflicted family member (assuring that this was a biologic relative), and the degree of the relative as defined above. When participants were unable to provide all of this information, cases were coded as not having FHD making it less likely that we would confirm our hypothesis that FHD was associated with worse NP testing. Participants who were adopted and could not provide information about their family history were excluded. All participants completed this portion of the assessment prior to completing the NP testing described below.

All participants completed a comprehensive NP battery designed to assess 7 different NP domains: verbal fluency, executive functions, speed of information processing, learning, recall, working memory, and motor skills. A list of each of the tests used to measure each of the NP domains is available in Table 1. This battery is the consensus NP battery for the CHARTER study and is widely used across other studies conducted at the HIV Neurobehavioral Research Center [46].

Raw NP test scores were converted into $T$-scores (standard scores with a mean of 50 and SD of 10) using demographically adjusted norms to control for the effects of age, education, gender, and where available ethnicity [47-49]. These demographically corrected $T$ scores were then summarized into a 0 - to 5-point deficit scale that places an emphasis on impaired performance, with anything greater than or equal to 0.5 indicating impairment. The deficit 
scores were derived using the following conversion: $T>39=0$ (no impairment); $T 35$ to 39 $=1$ deficit point; $T 30$ to $34=2$ points; $T 25$ to $29=3$ points; $T 20$ to $24=4$ points; $T<20=$ 5 points. Both domain deficit scores (DDS) and a global deficit score (GDS) were calculated by adding the deficit points of each contributing test, and dividing it by the number of tests per domain or over the whole battery [50-52]. A GDS score greater than or equal to 0.5 was used to define global NP impairment. DDS scores greater than 0.5 defined domain-specific NP impairment.

A comorbidity status defining the level of non-HIV-related contribution to NP impairment (i.e., incidental, contributing and confounding) was determined by a senior neuropsychologist (RKH) as described elsewhere [53]. We also assigned neurocognitive diagnoses using an algorithm derived from a published report yielding excellent interrater reliability in previous multisite studies [54]. This algorithm conforms to the Frascati criteria for diagnosing HAND [55] and requires, for a HIV-related neurocognitive diagnosis, the presence of mild impairment in at least two of the seven ability domains and the absence of significant confounding variables to be assigned, depending on severity of impairment and impact on everyday functioning, the following diagnoses were assigned: asymptomatic neurocognitive impairment (ANI), mild neurocognitive disorder (MND), and HIVassociated dementia (HAD) [55]. Persons with confounding comorbidity levels were assigned a diagnosis of neuropsychological impairment due to other causes (NPI-O).

\section{Analysis}

The comparison between the FHD and No FHD groups used Wilcoxon rank test for continuous variables (CD4 counts, estimated verbal IQ, GDS, DDS, etc), and Fisher's exact test for binary variables (ethnicity, NPI-O diagnosis, NP impairment, etc). Significance was defined when probability was less than a critical alpha of 0.05 .

\section{Results}

Table 2 shows that FHD positive and negative participants were similar in HIV diseaserelated and demographic factors, except that the FHD group had higher CD4 counts $(\mathrm{Z}=2.5$, $p=0.01$ ), higher estimated Verbal IQ (VIQ, $\mathrm{Z}=2.2, p=0.03$ ) and less ethnic diversity (Fisher's exact test, $p<0.01)$.

Despite having factors that are associated with better NP performance such as higher CD4 counts and estimated VIQ, participants with FHD had significantly worse NP ability than those without FHD as measured by the GDS (FHD mean=0.66 ( $\mathrm{SD}=0.64)$; No FHD mean $=0.55(\mathrm{SD}=0.56) ; \mathrm{Z}=2.0, p<0.05$, Cohen's $d=.18)($ Figure 1).

Potentially confounding neuropsychological comorbidities were unrelated to FHD. To assure that FHD and worse NP functioning were related in the least confounded individuals, we analyzed the relationship excluding those persons with confounding comorbidities. The FHD group still had worse global NP performance with a slightly stronger effect size (FHD mean $=0.59$ ( $\mathrm{SD}=0.61)$; No FHD mean=0.46 ( $\mathrm{SD}=0.46) ; \mathrm{Z}=2.1 ; p=0.03$, Cohen's $d=.24)$. An overall chi-square analysis, grouping persons into HAND (i.e., ANI, MND, and HAD) versus non-HAND (i.e., NP-normal) was significant (Fisher's exact test, $p<0.01$ ) with 53\% of the FHD group having a HAND diagnosis and 47\% of the No FHD group having a HAND diagnosis. Comparisons of individual HAND diagnoses by FHD versus No FHD were not significant.

Persons who reported first-degree relatives with dementia were no more likely to be NP impaired than those reporting second-degree relatives (person who reported both first and second degree relatives, were combined with those who reported first degree relatives). 
Eighty-seven percent (87\%) of participants in the FHD group reported 1 relative with dementia, $11 \%$ two relatives, $1 \%$ three relatives, $1 \%$ four relatives. A higher number of selfreported relatives with dementia was not associated with additional risk of NP impairment.

Analysis of impairment in each of the NP domains defined as DDS scores greater than 0.5 revealed that the FHD group was significantly more impaired than the No FHD group in the domains of verbal fluency, executive functions and motor skills (Figure 2).

\section{Discussion}

HAND appears to arise from multifactorial interactions of human immunity, neurologic factors, HIV invasion and replication in the CNS, and comorbidities. FHD appears to be a surrogate for genetic factors that increase the risk of HAND and in this study we observed worse neuropsychological functioning among HIV+ persons with FHD as compared to those without FHD.

NP impairments were found in the domains of verbal fluency, executive functioning and motor skills, cognitive capacities that are frequently damaged among HIV+ persons.

Executive impairments are consistent with damage to frontal subcortical structures. Verbal fluency impairments in HIV are likely due to difficulties with the executive components of the task (e.g., switching to a new strategy when the current strategy is exhausted) rather than language skills [56]. A defining feature of most dementias is memory impairment and we did not find differences in learning or memory between the FHD and No FHD groups in this study. This finding might suggest that family history of dementia lowers thresholds for the development of HIV-associated impairments and lessens the likelihood that these data represent a superimposed dementia on top of HIV-associated impairments. Clearly additional research is needed to tease apart the potential contributions of these factors for neurocognitive outcomes.

Synaptodendritic simplification in the frontal cortex has been shown to be associated with HIV-associated NP impairment [20, 21]. Furthermore, subtle motor impairments can develop as a result of damage to basal ganglia structures, which have also been documented to undergo HIV-associated synaptodendritic simplification and be associated with NP performance [23]. Damage to brain regions already affected by HIV and the associated NP impairments, may mean that there is increased susceptibility among those with FHD. A potential mechanism is that HIV accelerates abnormal protein aggregation frequently found in AD, HIV, and other dementias [11, 13]. Furthermore, individuals with FHD may have latent neurodegenerative conditions that HIV could accelerate by decreasing neuronal homeostasis.

Although family history had a small effect (Cohen's d .18 to .24) on level of NP impairment, it should be noted that our participants were relatively young (average age in mid 40s); it is possible that these effect sizes might be higher in older persons, consistent with the well recognized age dependency in evolution of neurocognitive decline related to neurodegenerative disease. As a post hoc analysis, we examined our participants to see if FHD had differential effects in older (i.e., $>50$ ) and younger $(<40)$ HIV+ individuals. Analysis did not reveal any significant effect of FHD on NP functioning in the younger or older groups.

It is curious that we saw a greater report of FHD among our Caucasian participants and those with significantly higher estimated VIQ scores and higher CD4 counts. All of these factors would typically be associated with better NP performance (although demographic corrections for ethnicity differences were employed); however, we observed the opposite, worse NP performance among those with FHD. One possibility is FHD participants with 
neurocognitive problems have become more aware of the cognitive problems of their family members and that the finding herein reflects this knowledge. Although possible, this hypothesis seems unlikely given that participants provided FHD information prior to formal NP testing in this study.

We have one of the largest sample sizes of any study of NP impairments in HIV+ persons; still, there are a few limitations. Ideally, a validated instrument could be used to diagnose FHD [45, 57], and corroborating evidence of FHD would have been sought from family members. Nevertheless, no incentives or disincentives to report FHD to the study are obvious. In future studies we will employ a standardized questionnaire of FHD that asks in a systematic way about the family history of various dementias and neurodegenerative conditions. We also plan to study the relationship of FHD with other possible covariates for HAND including genetic markers of dementia risk (e.g., APOE4).

Our study provides evidence that family history of dementia exerts a modest effect on likelihood that a person with HIV will have HAND. As the age of the population with HIV gradually increases with improved survival, FHD may be considered a convenient surrogate for one of several genetic risk factors for HAND. A positive screening for the presence of FHD may serve as a cue for clinicians to heighten awareness for potential HAND among their HIV-infected patients.

\section{Acknowledgments}

The CNS HIV Anti-Retroviral Therapy Effects Research (CHARTER) study is supported by award N01 MH22005 from the National Institutes of Health. The HIV Neurobehavioral Research Center (HNRC) is supported by Center award MH 62512 from NIMH.

\section{Appendix}

The San Diego HIV Neurobehavioral Research Center [HNRC] group is affiliated with the University of California, San Diego, the Naval Hospital, San Diego, and the Veterans Affairs San Diego Healthcare System, and includes: Director: Igor Grant, M.D.; CoDirectors: J. Hampton Atkinson, M.D., Ronald J. Ellis, M.D., Ph.D., and J. Allen McCutchan, M.D.; Center Manager: Thomas D. Marcotte, Ph.D.; Jennifer Marquie-Beck, M.P.H.; Melanie Sherman; Neuromedical Component: Ronald J. Ellis, M.D., Ph.D. (P.I.), J. Allen McCutchan, M.D., Scott Letendre, M.D., Edmund Capparelli, Pharm.D., Rachel Schrier, Ph.D., Terry Alexander, R.N., Debra Rosario, M.P.H., Shannon LeBlanc; Neurobehavioral Component: Robert K. Heaton, Ph.D. (P.I.), Steven Paul Woods, Psy.D., Mariana Cherner, Ph.D., David J. Moore, Ph.D.; Matthew Dawson; Neuroimaging Component: Terry Jernigan, Ph.D. (P.I.), Christine Fennema-Notestine, Ph.D., Sarah L. Archibald, M.A., John Hesselink, M.D., Jacopo Annese, Ph.D., Michael J. Taylor, Ph.D.; Neurobiology Component: Eliezer Masliah, M.D. (P.I.), Ian Everall, FRCPsych., FRCPath., Ph.D., Cristian L. Achim, M.D., Ph.D.; Neurovirology Component: Douglas Richman, M.D., (P.I.), David M. Smith, M.D.; International Component: J. Allen McCutchan, M.D., (P.I.); Developmental Component: Cristian L. Achim, M.D., Ph.D., Stuart Lipton, M.D., Ph.D.; Participant Accrual and Retention Unit: J. Hampton Atkinson, M.D. (P.I.), Rodney von Jaeger, M.P.H.; Data Management Unit: Anthony C. Gamst, Ph.D. (P.I.), Clint Cushman (Data Systems Manager); Statistics Unit: Ian Abramson, Ph.D. (P.I.), Florin Vaida, Ph.D., Reena Deutsch, Ph.D., Tanya Wolfson, M.A.

The CNS HIV Anti-Retroviral Therapy Effects Research (CHARTER) group is affiliated with the Johns Hopkins University, Mount Sinai School of Medicine, University of California, San Diego, University of Texas, Galveston, University of Washington, Seattle, Washington University, St. Louis and is headquartered at the University of California, San 
Diego and includes: Director: Igor Grant, M.D.; Co-Directors: J. Allen McCutchan, M.D., Ronald J. Ellis, M.D., Ph.D., Thomas D. Marcotte, Ph.D.; Center Manager: Donald Franklin, Jr.; Neuromedical Component: Ronald J. Ellis, M.D., Ph.D. (P.I.), J. Allen McCutchan, M.D., Terry Alexander, R.N.; Laboratory, Pharmacology and Immunology Component: Scott Letendre, M.D. (P.I.), Edmund Capparelli, Pharm.D.; Neurobehavioral Component: Robert K. Heaton, Ph.D. (P.I.), J. Hampton Atkinson, M.D., Steven Paul Woods, Psy.D., Matthew Dawson; Virology Component: Joseph K. Wong, M.D. (P.I.); Imaging Component: Christine Fennema-Notestine, Ph.D. (P.I.), Terry L., Jernigan, Ph.D., Michael J. Taylor, Ph.D., Rebecca Theilmann, Ph.D.; Data Management Unit: Anthony C. Gamst, Ph.D. (P.I.), Clint Cushman,; Statistics Unit: Ian Abramson, Ph.D. (P.I.), Florin Vaida, Ph.D.; Protocol Coordinating Component: Thomas D. Marcotte, Ph.D. (P.I.), Rodney von Jaeger, M.P.H.; Johns Hopkins University Site: Justin McArthur (P.I.), Mary Smith; Mount Sinai School of Medicine Site: Susan Morgello, M.D. (Co-P.I.) and David Simpson, M.D. (Co-P.I.), Letty Mintz, N.P.; University of California, San Diego Site: J. Allen McCutchan, M.D. (P.I.), Will Toperoff, N.P..; University of Washington, Seattle Site: Ann Collier, M.D. (Co-P.I.) and Christina Marra, M.D. (Co-P.I.), Trudy Jones, M.N., A.R.N.P.; University of Texas, Galveston Site: Benjamin Gelman, M.D., Ph.D. (P.I.), Eleanor Head, R.N., B.S.N.; and Washington University, St. Louis Site: David Clifford, M.D. (P.I.), Muhammad AlLozi, M.D., Mengesha Teshome, M.D.

The views expressed in this article are those of the authors and do not reflect the official policy or position of the Department of the Navy, Department of Defense, nor the United States Government.

\section{References}

1. Palella FJ Jr, et al. Mortality in the highly active antiretroviral therapy era: changing causes of death and disease in the HIV outpatient study. J Acquir Immune Defic Syndr. 2006; 43(1):27-34. [PubMed: 16878047]

2. Goodkin K, et al. Aging and neuro-AIDS conditions and the changing spectrum of HIV-1associated morbidity and mortality. J Clin Epidemiol. 2001; 54 Suppl 1:S35-S43. [PubMed: 11750208]

3. Effros RB, et al. Aging and infectious diseases: workshop on HIV infection and aging: what is known and future research directions. Clin Infect Dis. 2008; 47(4):542-553. [PubMed: 18627268]

4. Ferrando S, et al. Highly active antiretroviral treatment in HIV infection: benefits for neuropsychological function. AIDS. 1998; 12(8):F65-F70. [PubMed: 9631133]

5. Sacktor NC. Advances in the treatment of HIV dementia. AIDS Read. 1999; 9(1):57-60. 62. [PubMed: 12728886]

6. Tozzi V, et al. Positive and sustained effects of highly active antiretroviral therapy on HIV-1associated neurocognitive impairment. AIDS. 1999; 13(14):1889-1897. [PubMed: 10513647]

7. Suarez S, et al. Outcome of patients with HIV-1-related cognitive impairment on highly active antiretroviral therapy. AIDS. 2001; 15(2):195-200. [PubMed: 11216927]

8. Kohli R, et al. Aging and HIV infection. J Urban Health. 2006; 83(1):31-42. [PubMed: 16736353]

9. Grant I. Neurocognitive disturbances in HIV. Int Rev Psychiatry. 2008; 20(1):33-47. [PubMed: 18240061]

10. Cysique LA, Maruff P, Brew BJ. Prevalence and pattern of neuropsychological impairment in human immunodeficiency virus-infected/acquired immunodeficiency syndrome (HIV/AIDS) patients across pre- and post-highly active antiretroviral therapy eras: a combined study of two cohorts. J Neurovirol. 2004; 10(6):350-357. [PubMed: 15765806]

11. Achim CL, et al. Increased accumulation of intraneuronal amyloid beta in HIV-infected patients. J Neuroimmune Pharmacol. 2009; 4(2):190-199. [PubMed: 19288297]

12. Rempel HC, Pulliam L. HIV-1 Tat inhibits neprilysin and elevates amyloid beta. AIDS. 2005; 19(2):127-135. [PubMed: 15668537] 
13. Green DA, et al. Brain deposition of beta-amyloid is a common pathologic feature in HIV positive patients. AIDS. 2005; 19(4):407-411. [PubMed: 15750394]

14. Esiri MM, Biddolph SC, Morris CS. Prevalence of Alzheimer plaques in AIDS. J Neurol Neurosurg Psychiatry. 1998; 65(1):29-33. [PubMed: 9667557]

15. Jernigan TL, et al. Effects of methamphetamine dependence and HIV infection on cerebral morphology. Am J Psychiatry. 2005; 162(8):1461-1472. [PubMed: 16055767]

16. Ernst T, Chang L. Effect of aging on brain metabolism in antiretroviral-naive HIV patients. AIDS. 2004; 18 Suppl 1:S61-S67. [PubMed: 15075499]

17. Kaul M, Lipton SA. Chemokines and activated macrophages in HIV gp120-induced neuronal apoptosis. Proc Natl Acad Sci U S A. 1999; 96(14):8212-8216. [PubMed: 10393974]

18. Nath A. Human immunodeficiency virus (HIV) proteins in neuropathogenesis of HIV dementia. J Infect Dis. 2002; 186 Suppl 2:S193-S198. [PubMed: 12424697]

19. Garden GA, et al. Caspase cascades in human immunodeficiency virus-associated neurodegeneration. J Neurosci. 2002; 22(10):4015-4024. [PubMed: 12019321]

20. Everall IP, et al. Cortical synaptic density is reduced in mild to moderate human immunodeficiency virus neurocognitive disorder. HNRC Group. HIV Neurobehavioral Research Center. Brain Pathol. 1999; 9(2):209-217. [PubMed: 10219738]

21. Masliah E, et al. Dendritic injury is a pathological substrate for human immunodeficiency virusrelated cognitive disorders. HNRC Group. The HIV Neurobehavioral Research Center. Ann Neurol. 1997; 42(6):963-972. [PubMed: 9403489]

22. Bell JE. The neuropathology of adult HIV infection. Rev Neurol (Paris). 1998; 154(12):816-829. [PubMed: 9932303]

23. Moore DJ, et al. Cortical and subcortical neurodegeneration is associated with HIV neurocognitive impairment. AIDS. 2006; 20(6):879-887. [PubMed: 16549972]

24. Becker JT, et al. Prevalence of cognitive disorders differs as a function of age in HIV virus infection. AIDS. 2004; 18 Suppl 1:S11-S18. [PubMed: 15075493]

25. Larussa D, et al. Highly active antiretroviral therapy reduces the age-associated risk of dementia in a cohort of older HIV-1-infected patients. AIDS Res Hum Retroviruses. 2006; 22(5):386-392. [PubMed: 16706614]

26. Valcour V, et al. Age, apolipoprotein E4, and the risk of HIV dementia: the Hawaii Aging with HIV Cohort. J Neuroimmunol. 2004; 157(1-2):197-202. [PubMed: 15579298]

27. Valcour V, et al. Higher frequency of dementia in older HIV-1 individuals: the Hawaii Aging with HIV-1 Cohort. Neurology. 2004; 63(5):822-827. [PubMed: 15365130]

28. Valcour VG, et al. Cognitive impairment in older HIV-1-seropositive individuals: prevalence and potential mechanisms. AIDS. 2004; 18 Suppl 1:S79-S86. [PubMed: 15075502]

29. Barclay TR, et al. Age-associated predictors of medication adherence in HIV-positive adults: health beliefs, self-efficacy, and neurocognitive status. Health Psychol. 2007; 26(1):40-49. [PubMed: 17209696]

30. Hinkin $\mathrm{CH}$, et al. Medication adherence in HIV-infected adults: effect of patient age cognitive status, and substance abuse. AIDS. 2004; 18 Suppl 1:S19-S25. [PubMed: 15075494]

31. Hinkin $\mathrm{CH}$, et al. Medication adherence among HIV+ adults: effects of cognitive dysfunction and regimen complexity. Neurology. 2002; 59(12):1944-1950. [PubMed: 12499488]

32. Corder EH, et al. Gene dose of apolipoprotein E type 4 allele and the risk of Alzheimer's disease in late onset families. Science. 1993; 261(5123):921-923. [PubMed: 8346443]

33. Saunders AM, et al. Apolipoprotein E epsilon 4 allele distributions in late-onset Alzheimer's disease and in other amyloid-forming diseases. Lancet. 1993; 342(8873):710-711. [PubMed: 8103823]

34. Poirier J, et al. Apolipoprotein E polymorphism and Alzheimer's disease. Lancet. 1993; 342(8873): 697-699. [PubMed: 8103819]

35. Strittmatter WJ, et al. Apolipoprotein E: high-avidity binding to beta-amyloid and increased frequency of type 4 allele in late-onset familial Alzheimer disease. Proc Natl Acad Sci U S A. 1993; 90(5):1977-1981. [PubMed: 8446617] 
36. Mahley RW, Weisgraber KH, Huang Y. Apolipoprotein E4: a causative factor and therapeutic target in neuropathology, including Alzheimer's disease. Proc Natl Acad Sci U S A. 2006; 103(15):5644-5651. [PubMed: 16567625]

37. Feskens EJ, et al. Apolipoprotein e4 allele and cognitive decline in elderly men. BMJ. 1994; 309(6963):1202-1206. [PubMed: 7987151]

38. Henderson AS, et al. Apolipoprotein E allele epsilon 4, dementia, and cognitive decline in a population sample. Lancet. 1995; 346(8987):1387-1390. [PubMed: 7475820]

39. Jayadev S, et al. Conjugal Alzheimer disease: risk in children when both parents have Alzheimer disease. Arch Neurol. 2008; 65(3):373-378. [PubMed: 18332250]

40. Lautenschlager NT, et al. Risk of dementia among relatives of Alzheimer's disease patients in the MIRAGE study: What is in store for the oldest old? Neurology. 1996; 46(3):641-650. [PubMed: 8618660]

41. van Duijn CM, Stijnen T, Hofman A. Risk factors for Alzheimer's disease: overview of the EURODEM collaborative re-analysis of case-control studies. EURODEM Risk Factors Research Group. Int J Epidemiol. 1991; 20 Suppl 2:S4-S12. [PubMed: 1833353]

42. Fox NC, et al. Presymptomatic cognitive deficits in individuals at risk of familial Alzheimer's disease. A longitudinal prospective study. Brain. 1998; 121(Pt 9):1631-1639. [PubMed: 9762953]

43. Duara R, et al. Alzheimer's disease: interaction of apolipoprotein E genotype, family history of dementia, gender, education, ethnicity, and age of onset. Neurology. 1996; 46(6):1575-1579. [PubMed: 8649551]

44. Huang W, et al. APOE genotype, family history of dementia, and Alzheimer disease risk: a 6-year follow-up study. Arch Neurol. 2004; 61(12):1930-1934. [PubMed: 15596614]

45. Hayden KM, et al. Effects of family history and apolipoprotein E epsilon4 status on cognitive decline in the absence of Alzheimer dementia: the Cache County Study. Arch Neurol. 2009; 66(11):1378-1383. [PubMed: 19901170]

46. Cysique LA, Brew BJ. Neuropsychological functioning and antiretroviral treatment in HIV/AIDS: a review. Neuropsychol Rev. 2009; 19(2):169-185. [PubMed: 19424802]

47. Cherner M, et al. Demographically corrected norms for the Brief Visuospatial Memory Testrevised and Hopkins Verbal Learning Test-revised in monolingual Spanish speakers from the U.S.-Mexico border region. Arch Clin Neuropsychol. 2007; 22(3):343-353. [PubMed: 17293078]

48. Heaton, RK.; Taylor, MJ.; Manly, J. Demographic Effects and Use of Demographically Corrected Norms with the WAIS-III and WMS-III. In: David, ST., et al., editors. Clinical Interpretation of the WAIS-III and WMS-III. San Diego: Academic Press; 2003. p. 181-210.

49. Heaton, RK.; MS; Taylor, MJ.; Grant, I. Psychological Assessment Resources. 2004. Revised Comprehensive Norms for an Expanded Halstead-Reitan Battery: Demographically Adjusted Neuropsychological Norms for African American and Caucasian Adults.

50. Woods SP, et al. Executive dysfunction and neuropsychiatric symptoms predict lower health status in essential tremor. Cogn Behav Neurol. 2008; 21(1):28-33. [PubMed: 18327020]

51. Carey CL, et al. Predictive validity of global deficit scores in detecting neuropsychological impairment in HIV infection. J Clin Exp Neuropsychol. 2004; 26(3):307-319. [PubMed: 15512922]

52. Heaton RK, et al. The HNRC 500--neuropsychology of HIV infection at different disease stages. HIV Neurobehavioral Research Center. J Int Neuropsychol Soc. 1995; 1(3):231-251. [PubMed: 9375218]

53. Heaton RK, et al. HIV-associated neurocognitive disorders persist in the era of potent antiretroviral therapy. Neurology. 2010; 75(23):2087-2096. [PubMed: 21135382]

54 . Woods SP, et al. Interrater reliability of clinical ratings and neurocognitive diagnoses in HIV. J Clin Exp Neuropsychol. 2004; 26(6):759-778. [PubMed: 15370374]

55. Antinori A, et al. Updated research nosology for HIV-associated neurocognitive disorders. Neurology. 2007; 69(18):1789-1799. [PubMed: 17914061]

56. Iudicello JE, et al. Cognitive mechanisms of switching in HIV-associated category fluency deficits. J Clin Exp Neuropsychol. 2008; 30(7):797-804. [PubMed: 18608694]

57. Weissman MM, et al. Family-genetic studies of psychiatric disorders. Developing technologies. Arch Gen Psychiatry. 1986; 43(11):1104-1116. [PubMed: 3532996] 


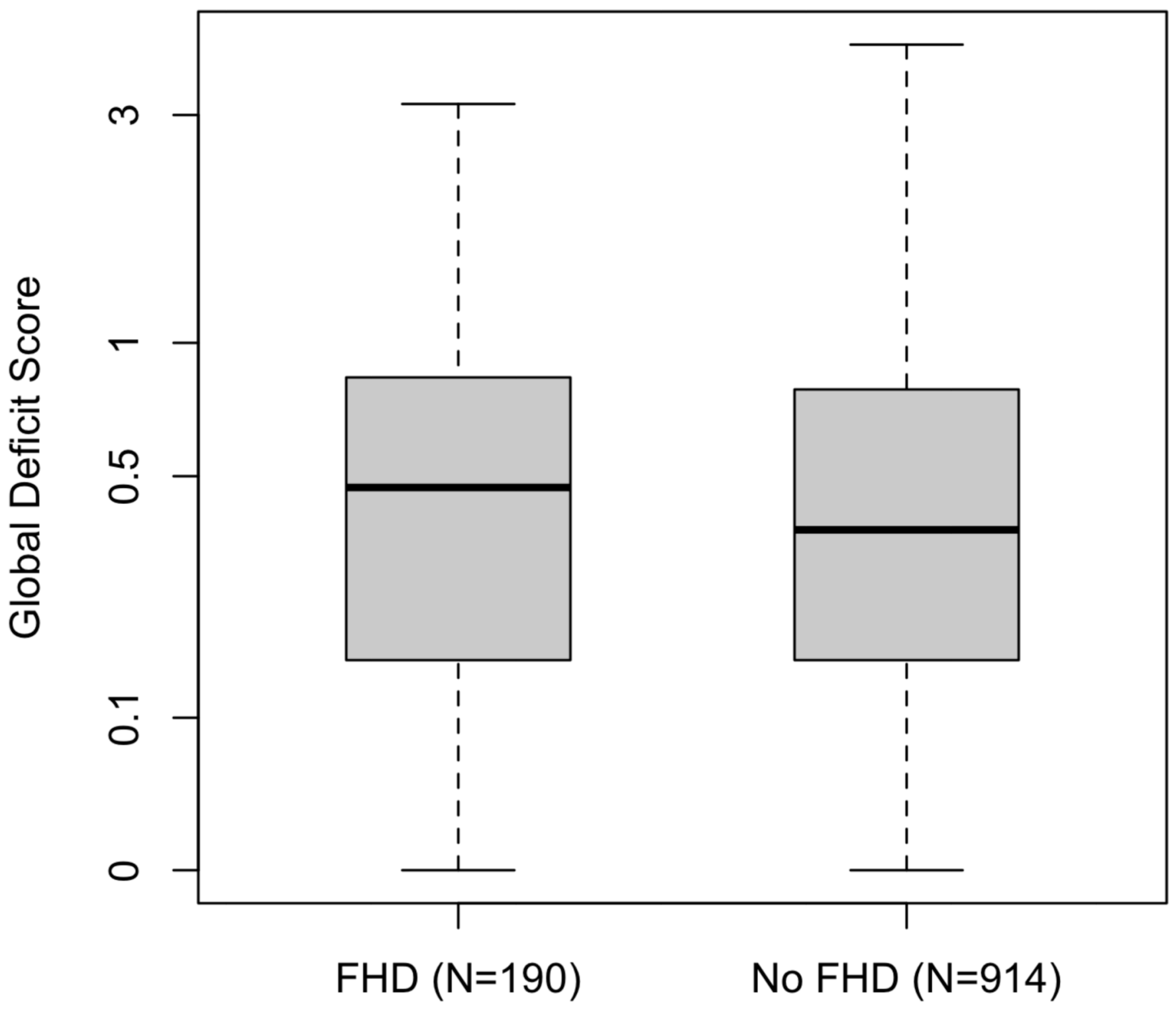

Figure 1.

HIV+ persons with a FHD have worse NP functioning that those without FHD, $\mathrm{p}=0.041$. The horizontal bars represent median GDS values, the boxes contain the middle $50 \%$ of the observations, and the whiskers extend to the minimum and maximum GDS values. The GDS is plotted on a logarithmic scale. 


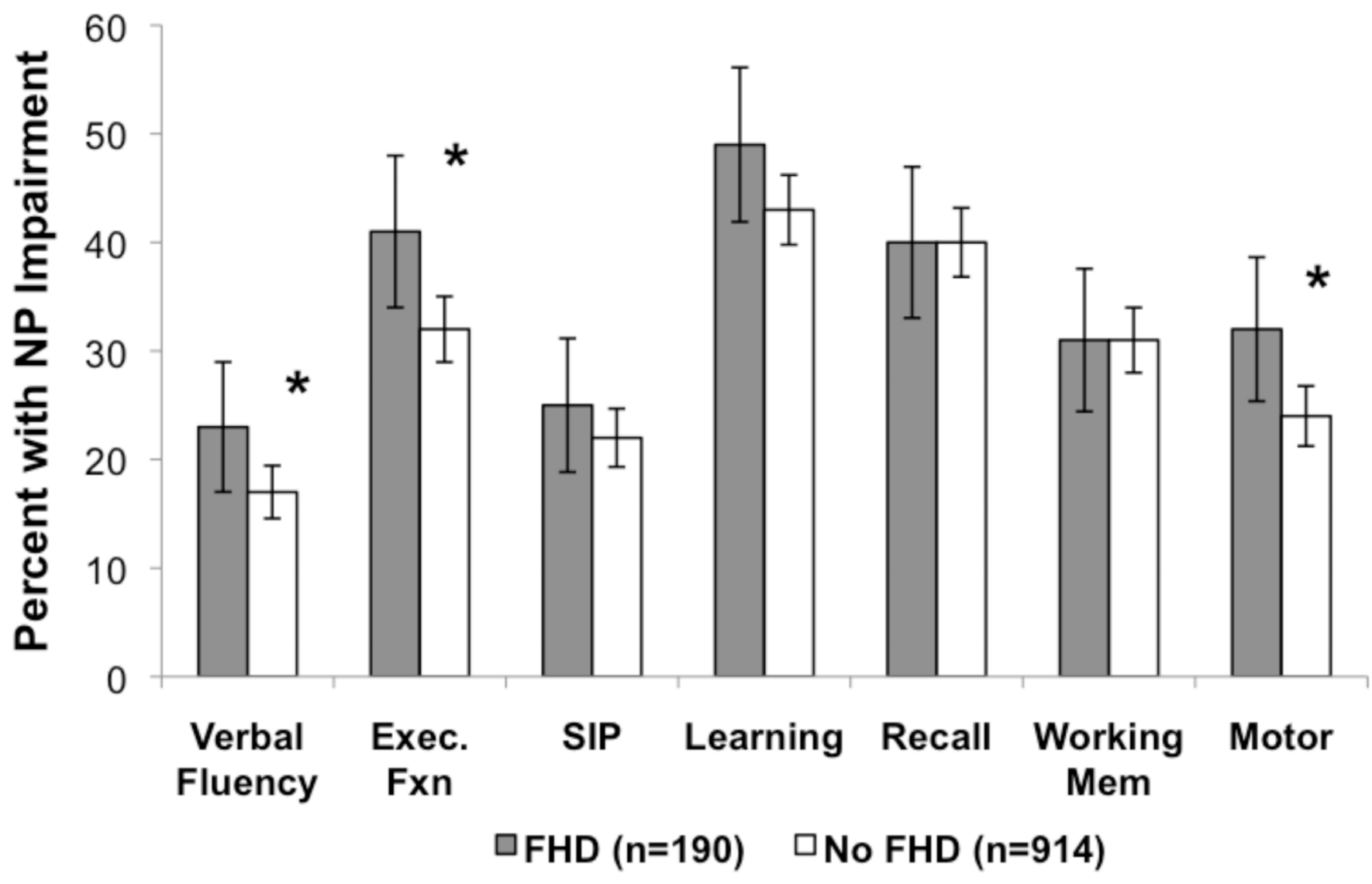

Figure 2.

More impairment was observed in Verbal Fluency, Executive Functions and Motor Skills among HIV+ persons with FHD. ${ }^{*} \mathrm{p}<0.05$; standard error bars represent $95 \%$ confidence intervals. 
Table 1

Neuropsychological (NP) tests by domain

\begin{tabular}{l}
\hline Verbal functioning \\
Letter Fluency \\
Category Fluency \\
Executive functioning \\
Trail Making Test Part B \\
Wisconsin Card Sorting Test-64 \\
Processing speed \\
Trail Making Test Part A \\
WAIS-III Digit Symbol \\
WAIS-III Symbol Search \\
Attention/working memory \\
Paced Auditory Serial Addition Test \\
WAIS-III Letter-Number Sequencing \\
Learning \\
Heaton Story Memory Test - learning \\
Heaton Figure Memory Test - learning \\
Hopkins Verbal Learning Test-Revised - Total Recall \\
Brief Visuospatial Memory Test-Revised - Total Recall \\
Memory \\
Heaton Story Memory Test - Delay \\
Heaton Figure Memory Test - Delay \\
Hopkins Verbal Learning Test-Revised - Delay Recall \\
Motor \\
\hline
\end{tabular}


Table 2

Demographics, HIV Disease, and Psychiatric characteristics by group.

\begin{tabular}{|c|c|c|}
\hline $\begin{array}{l}\text { Variable } \\
\text { Mean (SD) or \%(n) }\end{array}$ & $\underset{(n=190)}{\text { HIV+ FHD }}$ & $\begin{array}{c}\text { HIV+ No FHD } \\
(n=916)\end{array}$ \\
\hline \multicolumn{3}{|l|}{ Demographic Characteristics } \\
\hline Age (years) & $43.6(9.0)$ & $43.3(8.4)$ \\
\hline Education (years) & $12.9(2.6)$ & $12.5(2.5)$ \\
\hline Sex $(\%$ men $)$ & $73 \%(138)$ & $78.0 \%(711)$ \\
\hline Ethnicity (\% Caucasian) ${ }^{*}$ & $49 \%(93)$ & $36.0 \%(330)$ \\
\hline WRAT-III Est. verbal IQ* & $93.4(15.0)$ & $90.3(16.8)$ \\
\hline \multicolumn{3}{|l|}{ HIV Disease Characteristics } \\
\hline Current CD4 Count ${ }^{*}$ & $498(296)$ & $443(279)$ \\
\hline Nadir CD4 Count & $216(191)$ & $200.0(192)$ \\
\hline HIV RNA plasma $(\log 10)$ & $2.2(1.7,3.5)$ & $2.4(1.7,4.1)$ \\
\hline HIV RNA CSF $(\log 10)$ & $1.7(1.7,1.9)$ & $1.7(1.7,2.5)$ \\
\hline Proportion with AIDS & $61 \%(115)$ & $64 \%(582)$ \\
\hline Proportion with $\mathrm{HCV}$ & $23 \%(43)$ & $28 \%(251)$ \\
\hline \multicolumn{3}{|l|}{ Psychiatric Characteristics } \\
\hline BDI-II & $15.6(10.5)$ & $13.3(10.6)$ \\
\hline Any substance Abuse/Dep (lifetime) & $69 \%(130)$ & $74 \%(675)$ \\
\hline
\end{tabular}

